\title{
Prediction for the Mass Spectra of Resonance Mesons in the Soft-Wall AdS/QCD with a Modified 5D Metric
}

\author{
Yan-Qin Sui, Yue-Liang Wu, Zhi-Feng Xie and Yi-Bo Yang \\ Kavli Institute for Theoretical Physics China (KITPC) \\ Key Laboratory of Frontiers in Theoretical Physics \\ Institute of Theoretical Physics Chinese Academy of Sciences \\ Beijing, 100190, People's Republic of China
}

\begin{abstract}
A soft-wall anti-de Sitter/QCD model with a modified five-dimensional metric at the infrared region is constructed to obtain a nontrivial dilaton solution, which incorporates the chiral symmetry breaking and linear confinement. By taking the pion mass and decay constant as two input mass scales, the resulting predictions for the resonance states of pseudoscalar, scalar, vector and axialvector mesons agree remarkably with the experimentally confirmed resonance states. The effects of the quartic interaction term are investigated by taking an appropriate sign and magnitude for maintaining the stability of the bulk scalar potential. It is shown that such a simply modified soft-wall anti-de Sitter/QCD model can lead to a consistent prediction for the mass spectra of resonance states in the pseudoscalar, scalar, vector and axial-vector mesons; the agreement with the experimental data is found to be better than $10 \%$ for the excited meson states. The resulting pion form factor also agrees well with the experimental data.

PACS numbers: 12.40.-y,12.38.Aw,12.38.Lg,14.40.-n
\end{abstract}




\section{INTRODUCTION}

Strong interactions of quarks are described in the standard model by an $S U(3)$ gauge theory known as quantum chromodynamics (QCD) [1]. As the gauge group is non-Abelian, the gluons have direct self-interactions that lead to the well-known asymptotic freedom [2, 3] due to a negative beta function, $\beta(\mu)$, which causes the coupling constant $\alpha_{s}(\mu)$ to decrease at short distances (UV region), so that perturbative QCD at the UV region works well. At low energies (IR region), perturbative methods are no longer applicable as the coupling constant $\alpha_{s}(\mu)$ grows in the IR. We are currently unable to solve from first principle the low energy dynamics of QCD; one can then construct effective quantum field theories to describe the low energy features of QCD, such as dynamically generated spontaneous symmetry breaking [4]. It has been shown in Ref. [5] that such a dynamically generated spontaneous chiral symmetry breaking can lead to the consistent mass spectra for both the lowest lying nonet pseudoscalar mesons and nonet scalar mesons. Though the resulting mass spectra for the ground states were found to agree well with the experimental data, it is not manifest in a chiral effective field theory how to characterize the excited meson states.

It is well-known that there are two important features of QCD at the low energy: they are the chiral symmetry breaking and linear confinement. It was shown in [6] that for an $S U\left(N_{c}\right)$ QCD one may carry out a $1 / N_{c}$ expansion in a large $N_{c}$ limit. In this limit, theory remains maintaining the most important features such as color confinement and dynamical chiral symmetry breaking. Thus any consistent low energy QCD model should simultaneously characterize these two basic features. Besides that, the model should also practically be applicable to calculate the low energy quantities of QCD, such as the decay and coupling constants, the mass spectra of various meson resonances.

The duality between gravity and gauge theories conjectured by Maldacena [7] and further developed in [8, 9] has shed new light on solving the problem of strongly coupled gauge theories. Thus the anti-de Sitter/conformal field theory (AdS/CFT) conjecture is regarded as an important step in theoretical physics in the past ten years, which establishes the duality between the weak coupled supergravity in $A d S_{5}$ and the strong coupled $\mathcal{N}=4$ super YangMills, and thus makes the calculations in the strong coupled theory become feasible [10]. This feature has attracted a lot of attention recently. It is expected that an analogous duality holds between AdS and QCD, though the latter is not an exact conformal theory. There are two approaches to pursuing this duality: one is the so-called top-down approach [11] and the other is the bottom-up approach [12, 13]. The former starts with the string theory and varies the gravity background so as to reproduce the basic QCD features. The latter is inspired by the AdS/CFT conjecture and known as a phenomenological AdS/QCD model. The model consists of a gauge theory in a curved space (usually AdS) with the field contents chosen to holographically match some bound states and operators in QCD. It is also interesting to observe the correspondence between matrix elements obtained in AdS/CFT with the corresponding formula using the light-front representation as shown in 
Refs. [14 16].

The current impressive achievements of AdS/QCD models contain the chiral symmetry breaking in a hard-wall AdS/QCD model [12] and the linear confinement in a soft-wall AdS/QCD model [17]. Nevertheless, in the hard-wall model [12], the resulting mass spectra for the excited mesons are contrary to the experimental data. In the soft-wall model [17], one can obtain a desired mass spectra for the excited vector mesons, while the chiral symmetry breaking phenomenon cannot consistently be realized. Note that a dilaton field in the softwall model is introduced by hand as a uniform background field. Interesting progress was made in Refs. [18, 19]: a quartic interaction term in the bulk scalar potential was introduced to incorporate linear trajectories and chiral symmetry breaking. Nevertheless, such a term was shown [19] to cause an instability of the scalar potential and result in a negative mass for the lowest lying scalar meson state and much smaller mass spectra for other lowest lying meson states in comparison with the experimental data. Thus how to naturally incorporate these two important features into a single AdS/QCD model and obtain the consistent mass spectra remains a challenging and interesting task.

On this note, we provide an alternative soft-wall AdS/QCD model by simply modifying the five-dimensional (5D) metric in the IR region. The paper is organized as follows: In Sec. II, we introduce the modified 5D soft-wall AdS/QCD model and show how the background dilaton field gets a desired IR behavior from a simply modified 5D metric; several phenomenological AdS/QCD models corresponding to different choices of the bulk vacuum expectation value (VEV) of the scalar field are considered. In Sec. III, we provide a detailed analysis and show how such simply modified soft-wall AdS/QCD models can simultaneously describe both chiral symmetry breaking and linear confinement, and lead to a reasonable prediction for the mass spectra of the various resonance states in the pseudoscalar, scalar, vector and axial-vector mesons. Unlike the predictions given in [19], the simply modified AdS/QCD model in our present considerations contains no virtual meson state in the scalar sector. In particular, the resulting resonance meson states agree well with the experimentally confirmed meson states and there are no more additional unconfirmed resonance meson states existing in the present model. The effects of a quartic interaction term in the bulk scalar potential are investigated in Sec. IV. By taking an appropriate sign (that is opposite to the one considered in [19]) for the coupling constant of the quartic interaction to keep the stability of the bulk scalar potential, we found that the resulting predictions for the mass spectra of resonance mesons can be further improved and consistent with the experimental data. Our conclusions and remarks are presented in the last section.

\section{THE SOFT-WALL ADS/QCD MODEL WITH MODIFIED 5D METRIC}

In the real world, QCD is known to be neither supersymmetry nor quantum mechanically conformal. Therefore, the 5D space of AdS in the AdS/QCD model is not necessary to be 
a pure AdS. Here we shall consider the simplest extension to a 5D AdS with the following metric structure

$$
d s^{2}=a^{2}(z)\left(\eta_{\mu \nu} d x^{\mu} d x^{\nu}-d z^{2}\right) ; \quad a^{2}(z)=\left(1+\mu_{g}^{2} z^{2}\right) / z^{2}
$$

where $\eta_{\mu \nu}=\operatorname{diag}(1,-1,-1,-1)$, and $\mu_{g}$ is a constant mass scale. Such a nonpure AdS space was also considered in [20] with a hard-wall cut. Here we shall show that in the softwall AdS/QCD with the above simply modified 5D metric at the IR region, it can lead to a consistent prediction for the mass spectra in the pseudoscalar, scalar, vector and axial-vector meson resonances. The effects of a quartic term in the bulk scalar potential are found to further improve the mass spectra of resonance mesons when taking an appropriate sign and magnitude for the coupling constant.

It has been shown in [17] that the introduction of a background dilaton $\Phi$ can lead to a linear trajectory for resonance vector meson mass once $\Phi$ has an asymptotic behavior

$$
\Phi(z \rightarrow \infty)=\mu_{d}^{2} z^{2}
$$

where the parameter $\mu_{d}$ sets the meson mass scale. The 5D action with the background field of dilaton $\Phi(z)$ and a quartic term in the bulk scalar potential can be written as follows

$$
S_{5}=\int d^{5} x \sqrt{g} e^{-\Phi(z)} \operatorname{Tr}\left[|D X|^{2}-m_{X}^{2}|X|^{2}-\lambda|X|^{4}-\frac{1}{4 g_{5}^{2}}\left(F_{L}^{2}+F_{R}^{2}\right)\right]
$$

with $g=\left|\operatorname{det} g_{M N}\right|, D^{M} X=\partial^{M} X-i A_{L}^{M} X+i X A_{R}^{M}, A_{L, R}^{M}=A_{L, R}^{M a} t^{a}$ and $\operatorname{Tr}\left[t^{a} t^{b}\right]=\delta^{a b} / 2$. Here $A_{L, R}^{M}$ are introduced to gauge the chiral symmetry $S U(2)_{L} \times S U(2)_{R} \cdot \lambda$ is the coupling constant which has an opposite sign in comparison with the one in [19].

The parameter $g_{5}$ is fixed to be $g_{5}^{2}=12 \pi^{2} / N_{c}$ [12] with $N_{c}$ the color number and $m_{X}^{2}=-3$ by AdS/CFT correspondence. The UV boundary condition for the gauge fields $A_{L}$ and $A_{R}$ at $z=0$ is given by the value of the sources of the currents $J_{L}$ and $J_{R}$ in $4 \mathrm{D}$ theory as required by the holographic correspondence. The IR boundary condition with the background field dilaton playing the role of the smooth soft-wall cutoff simply requires that the action is finite at $z \rightarrow \infty$, so that the ambiguity of the choice of the IR boundary condition in a hard-wall theory [12] disappears in the soft-wall theory [17].

The VEV of $X$ field in 5D space has the following form for the two flavor case

$$
\langle X(z)\rangle=\frac{1}{2} v(z)\left(\begin{array}{ll}
1 & 0 \\
0 & 1
\end{array}\right)
$$

For simplicity, we shall first consider the case with $\lambda=0$. In this case, the VEV $v(z)$ satisfies the following condition

$$
\partial_{z}\left(a^{3}(z) e^{-\Phi} \partial_{z} v(z)\right)-a^{5}(z) e^{-\Phi} m_{X}^{2} v(z)=0 .
$$


For the role played by $X$ field on the boundary of $A d S_{5}[21]$, the VEV $v(z)$ has the following behavior at the UV boundary $z \rightarrow 0$ :

$$
v(z \rightarrow 0)=m_{q} \zeta z+\frac{\sigma z^{3}}{\zeta}
$$

where $m_{q}$ and $\sigma$ are interpreted by AdS/CFT duality as the quark mass and quark condensate respectively. The normalization $\zeta$ is fixed by QCD with $\zeta=\sqrt{3} /(2 \pi)$ [22]. The corresponding solution for the dilaton field at the UV boundary is found from Eq. (4) to be

$$
\Phi^{\prime}(z \rightarrow 0)=6 \mu_{g}^{2} z+O\left(z^{3}\right), \quad \Phi(z \rightarrow 0)=3 \mu_{g}^{2} z^{2}+O\left(z^{4}\right)
$$

The behavior of the VEV $v(z)$ in the IR boundary $z \rightarrow \infty$ is correlated to the dilaton behavior given in Eq. (2) which affects the mass spectra of meson resonances. In our present consideration with a modified metric given in Eq. (1), it is not difficult to find from Eq. (4) that a polynomial leading behavior for $v(z \rightarrow \infty)$ is enough to result in the required IR boundary condition for the dilaton background field. In general, we have the following IR boundary condition for $v(z)$

$$
v(z \rightarrow \infty)=\gamma\left(\mu_{d} z\right)^{\alpha}
$$

with $\alpha$ being a positive parameter. Instituting the above boundary condition into Eq. (4), we can obtain a solution for the dilaton at the IR boundary:

$$
\Phi^{\prime}(z \rightarrow \infty)=\frac{3 \mu_{g}^{2}}{\alpha} z, \quad \mu_{d}^{2}=\frac{3}{2} \mu_{g}^{2} / \alpha
$$

To investigate the dependence of the mass spectra of resonance mesons on the IR boundary conditions of the VEV $v(z)$, we are going to consider two interesting asymptotic behaviors of $v(z)$ at the IR boundary in three typical models which correspond to three different exact forms (I, II, III) of the VEV $v(z)$. One corresponds to $\alpha=1$ as shown in the models Ia, IIa, IIIa, and the other to $\alpha=\frac{1}{2}$ as shown in the models Ib, IIb, IIIb. Explicitly, two different asymptotic behaviors of $v(z)$ are given by

$$
\text { Case a : } \quad v(z \rightarrow \infty)=\gamma\left(\mu_{d} z\right) ; \quad \text { Case b }: \quad v(z \rightarrow \infty)=\gamma\left(\sqrt{\mu_{d} z}\right)
$$

The explicit forms of $v(z)$ for three types of models (I, II, III) with two IR boundary conditions are summarized in the Table II. The three quantities $m_{q}, \sigma$ and $\gamma$ appearing in the boundary conditions of $v(z)$ are mainly correlated to the three parameters $A, B$ and $C$ ( and $G$ in IIIb case). The model III was shown to be a well parametrized one in the modified soft-wall model [19]. It will be shown below that the results in our present considerations are not very sensitive to the exact forms of the bulk VEV $v(z)$; they mainly depend on the IR boundary conditions. For a comparison, we plot in Fig. 1 the bulk VEV $v(z)$ for two 


\begin{tabular}{ccc}
\hline \hline Models & $v(z)$ & Parameters \\
\hline Ia & $z\left(A+B z^{2}\right)\left(1+C z^{2}\right)^{-1}$ & $B=\frac{\sigma}{\zeta}+m_{q} \zeta C, C=B / \mu_{d} \gamma$ \\
Ib & $z\left(A+B z^{2}\right)\left(1+C z^{2}\right)^{-5 / 4}$ & $B=\frac{\sigma}{\zeta}+\frac{5}{4} m_{q} \zeta C, C=\left(B^{2} / \mu_{d} \gamma^{2}\right)^{2 / 5}$ \\
IIa & $z\left(A+B z^{2}\right)\left(1+C z^{4}\right)^{-1 / 2}$ & $B=\frac{\sigma}{\zeta}, C=\left(B / \mu_{d} \gamma\right)^{2}$ \\
IIb & $z\left(A+B z^{2}\right)\left(1+C z^{4}\right)^{-5 / 8}$ & $B=\frac{\sigma}{\zeta}, C=\left(B^{2} / \mu_{d} \gamma^{2}\right)^{4 / 5}$ \\
IIIa & $z\left[A+B \tanh \left(C z^{2}\right)\right]$ & $B=\mu_{d} \gamma-m_{q} \zeta, C=\frac{\sigma}{\zeta B}$ \\
IIIb & $z\left[A+B \tanh \left(C z^{2}\right)\right]\left(1+G z^{4}\right)^{-1 / 8}$ & $B=\mu_{d}^{1 / 2} \gamma G^{1 / 8}-m_{q} \zeta, C=\frac{\sigma}{\zeta B}$ \\
\hline \hline
\end{tabular}

TABLE I: Three type of models for $v(z)$ with two cases for each type of model and relevant parameters with $A=m_{q} \zeta$ for all the cases.

cases in model II. The corresponding dilaton field is plotted in Fig. 2 as the function of $z$. The three parameters $m_{q}, \sigma$ and $\gamma$ (or $A, B$ and $C$ ) are fixed by the known experimental values of $m_{\pi}=139.6 \mathrm{MeV}$ and $f_{\pi}=92.4 \mathrm{MeV}$ with minimizing the breaking of the GellMann-Oakes-Renner relation $f_{\pi}^{2} m_{\pi}^{2}=2 m_{q} \sigma$ at the $1 \%$ level. The parameter $G$ is obtained by optimizing the mass spectra of vector and axial-vector mesons in model IIIb.

The pion decay constant is calculated from the axial-vector equation of motion with the pole in the propagator set to zero which was discussed in detail in Ref. [12]. Note that the axial-vector equation of motion depends on both quark mass and condensate. The pion decay constant is given [12]:

$$
f_{\pi}^{2}=-\left.\frac{1}{g_{5}^{2}} \frac{\partial_{z} A(0, z)}{z}\right|_{z \rightarrow 0}
$$

where $A(0, z)$ is the axial-vector bulk-to-boundary propagator and is obtained by solving the equation of motion in the momentum space

$$
e^{\Phi} \partial_{z}\left(a(z) e^{-\Phi} \partial_{z} A(q, z)\right)+a(z) q^{2} A(q, z)-a^{3}(z) g_{5}^{2} v^{2}(z) A(q, z)=0
$$

with $q^{2}=0$ and the boundary conditions $A(0,0)=1$ and $\partial_{z} A(0, z \rightarrow \infty)=0$. The pion mass is related to the pseudoscalar equation of motion for the lowest lying state and will be discussed in Sec. \IIA.

\section{MASS SPECTRA OF PSEUDOSCALAR, SCALAR, VECTOR AND AXIAL- VECTOR MESONS}

In this section, we are going to make numerical calculations for the mass spectra of pseudoscalar, scalar, vector and axial-vector mesons. As $\mu_{d}$ or $\mu_{g}$ scales the mass spectra of meson resonances, it is not difficult to find out its value from a global fitting, the best value for the case $\lambda=0$ is found to be

$$
\mu_{d}=445 \mathrm{MeV} ; \quad \mu_{\mathrm{g}}=363 \mathrm{MeV} \quad(\text { Case a }), \quad \mu_{\mathrm{g}}=257 \mathrm{MeV} \quad(\text { Case b) }
$$


The values of three fitting parameters $m_{q}, \sigma$ and $\gamma$ are presented in the Table II. The mass spectra for the pseudoscalar, scalar, vector and axial-vector resonance mesons are given in Tables III, IV, V and VI. All the quoted experimental data are taken from the particle data group (PDG) [23]. It is seen that the resonance states agree well with the experimentally confirmed states and the resulting mass spectra are consistent with the experimental values, except for the ground states of the scalar and axial-vector mesons which have masses smaller than the experimental data. The effects of a quartic interaction term in the bulk scalar potential are going to be studied in the next section and shown to be necessary for further improving the mass spectra.

\begin{tabular}{ccccccc}
\hline \hline Parameter & Ia & Ib & IIa & IIb & IIIa & IIIb \\
\hline$m_{q}(\mathrm{MeV})$ & 4.16 & 4.64 & 4.44 & 4.07 & 4.98 & 4.25 \\
$\sigma^{\frac{1}{3}}(\mathrm{MeV})$ & 275 & 265 & 265 & 272 & 255 & 268 \\
$\gamma$ & 0.178 & 0.136 & 0.153 & 0.112 & 0.164 & 0.112 \\
\hline \hline
\end{tabular}

TABLE II: The parameters $m_{q}, \sigma$ and $\gamma$ for $\lambda=0$ in three type of models with two cases $a$ and $b$ corresponding to the IR boundary conditions given in Eq. (9).

\section{A. Pseudoscalar Mesons}

Writing the bulk scalar field as $X(x, z) \equiv(v(z) / 2+S(x, z)) e^{2 i \pi(x, z)}$ with $S(x, z)$ being the scalar meson field and $\pi(x, z)=\pi^{a}(x, z) t^{a}$ being the pseudoscalar meson field, and decomposing the axial field in terms of its transverse and longitudinal components, $A_{\mu}^{a}=$ $A_{\mu \perp}^{a}+\partial_{\mu} \phi^{a}$, we then obtain, from the action Eq. (3) , the following equation of motion in the $4 \mathrm{D}$ momentum space with the $A_{5}=0$ gauge:

$$
\begin{aligned}
& \partial_{z}\left(a(z) e^{-\Phi} \partial_{z} \phi^{a}\right)+g_{5}^{2} a^{3}(z) v^{2}(z) e^{-\Phi}\left(\pi^{a}-\phi^{a}\right)=0 \\
& q^{2} \partial_{z} \phi^{a}-g_{5}^{2} a^{2}(z) v^{2}(z) \partial_{z} \pi^{a}=0
\end{aligned}
$$

By eliminating the longitudinal component field $\phi$ from the above coupled equation Eq. (13), we obtain the following equation for the $\pi$ field

$$
-\partial_{z}^{2} \tilde{\pi}(q, z)+V_{\pi}(z) \tilde{\pi}(q, z)=q^{2} \tilde{\pi}(q, z),
$$

where $\tilde{\pi}(q, z) \equiv \partial_{z} \pi(q, z)$ and

$$
\begin{aligned}
V_{\pi}(z) & =g_{5}^{2} a^{2}(z) v^{2}(z)+\frac{\Phi^{\prime 2}+2 \Phi^{\prime \prime}}{4}+\frac{15 a^{\prime 2}(z)}{4 a^{2}(z)}-\frac{3 a^{\prime}(z)\left(v(z) \Phi^{\prime}-2 v^{\prime}(z)\right)}{2 a(z) v(z)}-\frac{3 a^{\prime \prime}(z)}{2 a(z)} \\
& +\frac{2 v^{\prime 2}(z)}{v^{2}(z)}-\frac{\Phi^{\prime} v^{\prime}(z)+v^{\prime \prime}(z)}{v(z)} .
\end{aligned}
$$


Assuming $\tilde{\pi}(q, z)=\sum_{n} \Pi_{n}(q) \tilde{\pi}_{n}(z)$, we arrive at the following equation of motion

$$
-\partial_{z}^{2} \tilde{\pi}_{n}(z)+V_{\pi}(z) \tilde{\pi}_{n}(z)=m_{\pi_{n}}^{2} \tilde{\pi}_{n}(z)
$$

where $q^{2}$ is replaced by $m_{\pi_{n}}^{2}$ with $m_{\pi_{n}}$ being the masses of pseudoscalar mesons. The above equation can be solved by the shooting method. Using the boundary conditions $\tilde{\pi}(z \rightarrow 0)=0, \partial_{z} \tilde{\pi}(z \rightarrow \infty)=0$, we obtain the mass spectra of excited states with the input of $\pi$ mass and decay constant. The numerical results are given in Table. III and also plotted in Fig. 3. Where the mass of pion meson is as an input, the resulting excited meson states

\begin{tabular}{lccccccc}
\hline \hline $\mathrm{n} \pi$ experimental. $(\mathrm{MeV})$ & $\mathrm{Ia}$ & $\mathrm{Ib}$ & $\mathrm{IIa}$ & $\mathrm{IIb}$ & IIIa & IIIb \\
\hline 0 & 139.6 & 139.6 & 139.6 & 139.6 & 139.6 & 139.6 & 139.6 \\
1 & $1350 \pm 100$ & 1219 & 1127 & 1285 & 1474 & 1339 & 1524 \\
2 & $1816 \pm 14$ & 1632 & 1483 & 1664 & 1733 & 1721 & 1779 \\
3 & - & 1949 & 1754 & 1960 & 1958 & 2015 & 1999 \\
4 & - & 2218 & 1983 & 2212 & 2169 & 2264 & 2207 \\
5 & - & 2455 & 2185 & 2444 & 2388 & 2491 & 2422 \\
6 & - & 2670 & 2368 & 2677 & 2627 & 2717 & 2652 \\
7 & - & 2869 & 2536 & 2923 & 2881 & 2956 & 2897 \\
\hline \hline
\end{tabular}

TABLE III: The experimental and predicted mass spectra for pseudoscalar mesons with $\lambda=0$.

agree well with the data in models IIb, IIIa and IIIb. The agreement is seen to be within $10 \%$; it is then interesting to provide a prediction for possible high excited states.

\section{B. Scalar Mesons}

Assuming $X(x, z) \equiv(v(z) / 2+S(x, z)) e^{2 i \pi(x, z)}$ and $S(x, z)=\sum_{n} \mathcal{S}_{n}(x) S_{n}(z)$, we arrive at the following equation of motion

$$
\partial_{z}\left(a^{3}(z) e^{-\Phi} \partial_{z} S_{n}(z)\right)-a^{5}(z) e^{-\Phi} m_{X}^{2} S_{n}(z)=-a^{3}(z) e^{-\Phi} m_{S_{n}}^{2} S_{n}(z)
$$

By defining $S_{n}(z) \equiv e^{\omega_{s} / 2} s_{n}(z)=e^{(\Phi-3 \log a(z)) / 2} s_{n}(z)$, we have

$$
-\partial_{z}^{2} s_{n}(z)+\left(\frac{1}{4} \omega_{s}^{\prime 2}-\frac{1}{2} \omega_{s}^{\prime \prime}+a^{2}(z) m_{X}^{2}\right) s_{n}(z)=m_{S_{n}}^{2} s_{n}(z)
$$

For simplicity, we consider here only the mass spectra for the $S U(3)$ singlet scalar mesons which have more experimental data.

Using the shooting method to solve Eq. (18) with the boundary conditions $s_{n}(z \rightarrow 0)=0$, $\partial_{z} s_{n}(z \rightarrow \infty)=0$, we obtain the mass spectra for the $S U(3)$ singlet resonance scalar mesons which are given in Table IV and also plotted in Fig. [3. 


\begin{tabular}{lccccccc}
\hline \hline $\mathrm{n} f_{0}$ & experimental. $(\mathrm{MeV})$ & $\mathrm{Ia}$ & $\mathrm{Ib}$ & IIa & IIb & IIIa & IIIb \\
\hline 0 & $550_{-150}^{+250}$ & 115 & 121 & 119 & 127 & 115 & 126 \\
1 & $1350 \pm 150$ & 1002 & 1050 & 1099 & 1434 & 1122 & 1485 \\
2 & $1724 \pm 7$ & 1366 & 1418 & 1446 & 1697 & 1468 & 1743 \\
3 & $1992 \pm 16$ & 1644 & 1693 & 1713 & 1923 & 1734 & 1964 \\
4 & $2189 \pm 13$ & 1877 & 1925 & 1939 & 2124 & 1958 & 2158 \\
5 & - & 2083 & 2128 & 2140 & 2306 & 2155 & 2333 \\
6 & - & 2268 & 2312 & 2321 & 2474 & 2333 & 2494 \\
7 & - & 2440 & 2482 & 2489 & 2630 & 2497 & 2645 \\
\hline \hline
\end{tabular}

TABLE IV: The experimental and predicted mass spectra for the singlet scalar mesons with $\lambda=0$.

It is seen that the resonance states agree well with the experimentally confirmed states though the ground state has a small mass (about $120 \mathrm{MeV}$ ) in comparison with the experimental data $550_{-150}^{+250} \mathrm{MeV}$, which have the biggest uncertainties. Note that this is unlike the model only considering a quartic interaction in the bulk scalar potential [19], where it was shown that the model may cause an instability and contains virtual mass for the lowest lying scalar meson. This is avoided in the present modified models which contain no virtual mass state. It must be clarified that the scalar states $f_{0}(980 \pm 10), f_{0}(1505 \pm 6), f_{0}(2103 \pm 8)$ and $f_{0}(2314 \pm 25)$ should be classified into the isosinglet resonance scalar states of $S U(3)$ octet mesons, rather than the $S U(3)$ singlet resonance scalar states. As an interesting check, we plot in Fig. 4 the corresponding bulk wave functions of $S U(3)$ singlet resonance scalar mesons; it is seen that the oscillation property becomes manifest in the scalar sector. Note that the possible instanton effects and the mixing effects between the $S U(3)$ singlet scalar and the isosinglet scalar of the $S U(3)$ octet are not included in the present considerations. It is manifest from Fig. 3 that the models IIb and IIIb lead to a better agreement with the experimental data.

\section{Vector Mesons}

From the action Eq. (3), with the gauge fixing $V_{5}=0$, one can derive the equation of motion for vector field

$$
-\partial_{z}^{2} V_{n}+\omega^{\prime} \partial_{z} V_{n}=m_{V_{n}}^{2} V_{n}
$$

For simplicity, we omit the Lorentz index and group index in flavor space. Defining $V_{n} \equiv$ $e^{\omega / 2} v_{n}=e^{(\Phi(z)-\log a(z)) / 2} v_{n}$, the above equation can be rewritten as

$$
-\partial_{z}^{2} v_{n}+\left(\frac{1}{4} \omega^{\prime 2}-\frac{1}{2} \omega^{\prime \prime}\right) v_{n}=m_{V_{n}}^{2} v_{n} .
$$


Such an eigenvalue equation can also be solved by the shooting method, using the boundary conditions $v_{n}(z \rightarrow 0)=0, \partial_{z} v_{n}(z \rightarrow \infty)=0$; the resulting mass spectra are presented in Table. $\nabla$ and also plotted in Fig. 3. It is interesting to note that such a simple model can lead to a remarkable agreement with the experimental data, especially in the models IIb and IIIb. For an illustration, we also plot in Fig. 5 the bulk wave functions of the ground state and the excited state $(n=4)$ for various cases.

\begin{tabular}{lccccccc}
\hline \hline $\mathrm{n} \rho$ experimental. $(\mathrm{MeV})$ & $\mathrm{Ia}$ & $\mathrm{Ib}$ & IIa & IIb & IIIa & IIIb \\
\hline 0 & $775.5 \pm 1$ & 739 & 603 & 777 & 727 & 775 & 748 \\
1 & $1465 \pm 25$ & 1223 & 1175 & 1292 & 1468 & 1303 & 1501 \\
2 & $1720 \pm 20$ & 1534 & 1509 & 1596 & 1744 & 1610 & 1773 \\
3 & $1909 \pm 30$ & 1784 & 1769 & 1842 & 1971 & 1856 & 1999 \\
4 & $2149 \pm 17$ & 2000 & 1990 & 2054 & 2170 & 2068 & 2196 \\
5 & $2265 \pm 40$ & 2193 & 2187 & 2249 & 2351 & 2255 & 2373 \\
6 & - & 2370 & 2367 & 2417 & 2516 & 2426 & 2535 \\
7 & - & 2534 & 2532 & 2578 & 2671 & 2584 & 2685 \\
\hline \hline
\end{tabular}

TABLE V: The experimental and predicted mass spectra for vector mesons with $\lambda=0$.

\section{Axial-vector Mesons}

From the action Eq. (3) with the gauge $A_{5}=0$, one can derive the equation of motion for perpendicular component of axial field

$$
e^{\Phi} \partial_{z}\left(a(z) e^{-\Phi} \partial_{z} A_{n}\right)+a(z) q^{2} A_{n}-a^{3}(z) g_{5}^{2} v^{2}(z) A_{n}=0
$$

Again defining $A_{n} \equiv e^{\omega / 2} a_{n}=e^{(\Phi(z)-\log a(z)) / 2} a_{n}$, the above equation of motion can be reexpressed as

$$
-\partial_{z}^{2} a_{n}+\left(\frac{1}{4} \omega^{\prime 2}-\frac{1}{2} \omega^{\prime \prime}+g_{5}^{2} v^{2}(z) a^{2}(z)\right) a_{n}=m_{A_{n}}^{2} a_{n} .
$$

With the boundary conditions $a_{n}(z \rightarrow 0)=0, \partial_{z} a_{n}(z \rightarrow \infty)=0$, the resulting mass spectra by using the shooting method is given in Table.VI and also plotted in Fig. 3, The resonance states agree well with the experimental ones, while the mass for the ground state is slightly smaller than the experimental data. 


\begin{tabular}{lccccccc}
\hline \hline $\mathrm{n} a_{1}$ experimental. $(\mathrm{MeV})$ & $\mathrm{Ia}$ & $\mathrm{Ib}$ & IIa & IIb & IIIa & IIIb \\
\hline 0 & $1230 \pm 40$ & 934 & 714 & 940 & 807 & 963 & 833 \\
1 & $1647 \pm 22$ & 1468 & 1247 & 1496 & 1507 & 1539 & 1540 \\
2 & $1930_{-70}^{+30}$ & 1822 & 1573 & 1831 & 1778 & 1880 & 1807 \\
3 & $2096 \pm 122$ & 2109 & 1829 & 2102 & 2003 & 2152 & 2031 \\
4 & $2270_{-40}^{+55}$ & 2358 & 2049 & 2338 & 2202 & 2386 & 2228 \\
5 & - & 2582 & 2247 & 2549 & 2380 & 2594 & 2403 \\
6 & - & 2787 & 2439 & 2742 & 2545 & 2785 & 2564 \\
7 & - & 2979 & 2638 & 2922 & 2699 & 2964 & 2713 \\
\hline \hline
\end{tabular}

TABLE VI: The experimental and predicted mass spectra for axial-vectors with $\lambda=0$.

\section{QUARTIC INTERACTION OF BULK SCALAR}

It is interesting to note that the above simplest AdS/QCD model with four parameters can lead to a consistent prediction for all the experimentally confirmed resonance meson states, while the ground state masses of scalar and axial-vector mesons obtained above appear to be smaller than the experiment data though the lowest lying scalar mass has the biggest uncertainty. To make a possible improvement, we now turn to consider the effects of the quartic interaction $\lambda|X|^{4}$ in the bulk scalar potential. In this case, the equation for the $\operatorname{VEV} v(z)$ is modified to be

$$
\partial_{z}\left(a^{3}(z) e^{-\Phi} \partial_{z} v(z)\right)-a^{5}(z) e^{-\Phi}\left(m_{X}^{2}+\frac{\lambda}{2} v^{2}(z)\right) v(z)=0
$$

and the equation of motion for the scalar field is extended to be

$$
\partial_{z}\left(a^{3}(z) e^{-\Phi} \partial_{z} S_{n}(z)\right)-a^{5}(z) e^{-\Phi}\left(m_{X}^{2}+\frac{3}{2} \lambda v^{2}(z)\right) S_{n}(z)=-a^{3}(z) e^{-\Phi} m_{S_{n}}^{2} S_{n}(z)
$$

Using the shooting method and making a global fitting with input mass scales of the $\pi$ meson mass and decay constant as well as the Gell-Mann-Oakes-Renner relation, we present all the numerical results in Tables VIII, IX, X, XI and also plot them in Fig. 6. The model parameters are reanalyzed and given in Table VII. The reasonable value for the coupling constant $\lambda$ is found to be $\lambda=9$ and the parameter $G$ in model IIIb is fitted to be $G=0.01 \mathrm{GeV}^{4}$.

It is interesting to see that the inclusion of the quartic interaction term with an appropriate sign and magnitude can further improve the predictions. Especially, the models IIb and IIIb lead to a better agreement, which shows that the IR boundary condition

$v(z \rightarrow \infty) \sim \sqrt{z}$ is more reasonable than the IR boundary condition $v(z \rightarrow \infty) \sim z$. 


\begin{tabular}{ccccc}
\hline \hline Parameter & IIa & IIb & IIIa & IIIb \\
\hline$m_{q}(\mathrm{MeV})$ & 6.95 & 6.79 & 7.08 & 6.49 \\
$\sigma^{\frac{1}{3}}(\mathrm{MeV})$ & 228 & 229 & 226 & 233 \\
$\gamma$ & 0.30 & 0.20 & 0.29 & 0.20 \\
$\mu_{d}(\mathrm{MeV})$ & 412 & 548 & 412 & 557 \\
\hline \hline
\end{tabular}

TABLE VII: The fitting parameters $m_{q}, \sigma, \gamma, \mu_{d}$ with $\lambda=9$.

\begin{tabular}{lccccc}
\hline \hline $\mathrm{n} \pi$ & experimental. $(\mathrm{MeV})$ & $\mathrm{IIa}$ & $\mathrm{IIb}$ & $\mathrm{IIIa}$ & $\mathrm{IIIb}$ \\
\hline 0 & 139.6 & 139.6 & 139.6 & 139.6 & 139.6 \\
1 & $1350 \pm 100$ & 1399 & 1526 & 1441 & 1437 \\
2 & $1816 \pm 14$ & 1709 & 1871 & 1722 & 1824 \\
3 & - & 1979 & 2103 & 1975 & 2080 \\
4 & - & 2242 & 2295 & 2231 & 2283 \\
5 & - & 2500 & 2493 & 2488 & 2482 \\
6 & - & 2757 & 2716 & 2743 & 2704 \\
7 & - & 3013 & 2960 & 2998 & 2948 \\
\hline \hline
\end{tabular}

TABLE VIII: The experimental and predicted mass spectra for pseudoscalar mesons with $\lambda=9$.

\begin{tabular}{lccccc}
\hline \hline $\mathrm{n} f_{0}$ & experimental. $(\mathrm{MeV})$ & $\mathrm{IIa}$ & $\mathrm{IIb}$ & IIIa & IIIb \\
\hline 0 & $550_{-150}^{+250}$ & 493 & 317 & 495 & 305 \\
1 & $1350 \pm 150$ & 1083 & 1401 & 1100 & 1307 \\
2 & $1724 \pm 7$ & 1402 & 1757 & 1413 & 1708 \\
3 & $1992 \pm 16$ & 1707 & 1986 & 1702 & 1964 \\
4 & $2189 \pm 13$ & 1997 & 2158 & 1982 & 2154 \\
5 & - & 2278 & 2290 & 2260 & 2297 \\
6 & - & 2550 & 2373 & 2531 & 2392 \\
7 & - & 2815 & 2434 & 2793 & 2448 \\
\hline \hline
\end{tabular}

TABLE IX: The experimental and predicted mass spectra for scalar mesons with $\lambda=9$.

\section{VECTOR COUPLING AND PION FORM FACTOR}

As a useful check for the consistency of our present AdS/QCD model, we are going to perform a calculation for the vector coupling $g_{\rho \pi \pi}$ and the pion form factor $F_{\pi}\left(q^{2}\right)$. The 


\begin{tabular}{lccccc}
\hline \hline $\mathrm{n} \rho$ & experimental. $(\mathrm{MeV})$ & IIa & IIb & IIIa & IIIb \\
\hline 0 & $775.5 \pm 1$ & 583 & 646 & 584 & 661 \\
1 & $1465 \pm 25$ & 900 & 1468 & 893 & 1378 \\
2 & $1720 \pm 20$ & 1248 & 1793 & 1252 & 1753 \\
3 & $1909 \pm 30$ & 1564 & 2008 & 1565 & 1994 \\
4 & $2149 \pm 17$ & 1860 & 2170 & 1847 & 2174 \\
5 & $2265 \pm 40$ & 2143 & 2289 & 2122 & 2332 \\
6 & - & 2417 & 2353 & 2395 & 2372 \\
7 & - & 2685 & 2420 & 2661 & 2432 \\
\hline \hline
\end{tabular}

TABLE X: The experimental and predicted mass spectra for vector mesons with $\lambda=9$.

\begin{tabular}{lccccc}
\hline \hline $\mathrm{n} a_{1}$ & experimental$(\mathrm{MeV})$ & IIa & IIb & IIIa & IIIb \\
\hline 0 & $1230 \pm 40$ & 1128 & 913 & 1150 & 906 \\
1 & $1647 \pm 22$ & 1643 & 1618 & 1668 & 1544 \\
2 & $1930_{-70}^{+30}$ & 1953 & 1940 & 1950 & 1903 \\
3 & $2096 \pm 122$ & 2225 & 2161 & 2213 & 2146 \\
4 & $2270_{-40}^{+55}$ & 2486 & 2333 & 2473 & 2332 \\
5 & - & 2742 & 2470 & 2726 & 2480 \\
6 & - & 2993 & 2574 & 2974 & 2594 \\
7 & - & 3239 & 2642 & 3217 & 2667 \\
\hline \hline
\end{tabular}

TABLE XI: The experimental and predicted mass spectra for axial-vector mesons with $\lambda=9$.

vector coupling $g_{\rho \pi \pi}$ in the soft-wall AdS/QCD is given as follows[12]:

$$
g_{\rho \pi \pi}=\frac{g_{5}}{N} \int d z V_{\rho}(z) e^{-\Phi(z)}\left(\frac{a(z)\left(\partial_{z} \varphi(z)\right)^{2}}{g_{5}^{2}}+v^{2}(z) a^{3}(z)(\pi(z)-\varphi(z))^{2}\right)
$$

where $V_{\rho}(z)$ is the $\rho$ meson bulk wave function corresponding to the vector meson bulk wave functions $V_{n}$ with $n=0$. Note that in all the integrals over $z$, the integration region is in principle the whole range $z \in(0, \infty)$. While in the practical calculations, for the lower limit, one can set a value as small as possible so as to obtain a stable result, for the upper limit, considering the suppressed factor $e^{-\Phi(z)}$, one can take at a certain finite value where the integrand tends to be zero. $V_{n}$ satisfies the normalization condition:

$$
\int d z a(z) e^{-\Phi(z)} V_{n}(z) V_{m}(z)=\delta_{m n} .
$$

The functions $\pi(z)$ and $\varphi(z)$ are the solutions of Eq. (13) and normalized as follows:

$$
N=\int d z e^{-\Phi(z)}\left(\frac{a(z)\left(\partial_{z} \varphi(z)\right)^{2}}{g_{5}^{2}}+v^{2}(z) a^{3}(z)(\pi(z)-\varphi(z))^{2}\right)
$$


The numerical results are found to be $g_{\rho \pi \pi}=3.63 \mathrm{MeV}$ (IIa), $2.94 \mathrm{MeV}$ (IIb), $3.81 \mathrm{MeV}$ (IIIa), $3.12 \mathrm{MeV}$ (IIIb) with $\lambda=0$. After including the quartic interaction with $\lambda=9$, the results are changed to be $g_{\rho \pi \pi}=2.86 \mathrm{MeV}$ (IIa), $3.51 \mathrm{MeV}$ (IIb), $2.93 \mathrm{MeV}$ (IIIa), $3.53 \mathrm{MeV}$ (IIIb), which are still below the experimental value $g_{\rho \pi \pi}=6.03 \pm 0.07 \mathrm{MeV}$. Other soft-wall models also obtained small values, for instance, $g_{\rho \pi \pi}=2.89$ in [19].

We now carry out a calculation for the spacelike pion form factor $F_{\pi}\left(q^{2}\right)$ by adopting the expressions in terms of the vector and axial-vector bulk-to-boundary propagators as given in [24]:

$$
F_{\pi}\left(q^{2}\right)=\frac{g_{5}}{N} \int d z V(q, z) e^{-\Phi(z)}\left(\frac{a(z)\left(\partial_{z} \varphi(z)\right)^{2}}{g_{5}^{2}}+v^{2}(z) a^{3}(z)(\pi(z)-\varphi(z))^{2}\right)
$$

where $V(q, z)$ is the vector bulk-to-boundary propagator which satisfies the following equation

$$
-\partial_{z}^{2} V(q, z)+\omega^{\prime} \partial_{z} V(q, z)=q^{2} V(q, z)
$$

with the boundary condition $V(q, z \rightarrow 0)=1$, so that $V(q, z)$ only depends on $q^{2}$. The general considerations and derivations for the electromagnetic form factor in the AdS space can be seen in literature [25, 26], which may be compared with the corresponding light-front form given in 14 16]. Our result is plotted in Fig. 7 which shows a good agreement with the experimental data. It appears to be better than the one obtained in the AdS/QCD models considered in [19] and [24]. We would like to point out that though the coupling constant $g_{\rho \pi \pi}$ in Eq. (25) and the form factor $F_{\pi}$ in Eq. (28) have a similar expression, the latter agrees well with the experimental data and the former has a discrepancy of about a factor of 2 with the experimental result. The reason is that the form factor is obtained by considering contributions from all resonance mesons rather than only from the ground state $\rho$ meson, which may explicitly be seen from the following expression [24],

$$
F_{\pi}\left(q^{2}\right)=-\sum_{n=1}^{\infty} \frac{f_{n} g_{n \pi \pi}}{q^{2}-M_{n}^{2}}
$$

where the summation is over the ground state $\rho$ meson and its excited states, $f_{n}$ are related to their decay constants, $g_{n \pi \pi}$ are the corresponding coupling constants and $M_{n}$ are their masses. It is noticed that the pion form factor is not sensitive to the IR boundary conditions, but it is relevant to the magnitude of quartic interaction. Thus more precise experimental measurement may be used to determine the coupling constant $\lambda$.

\section{CONCLUSION}

In this paper, we have shown how the chiral symmetry breaking and linear confinement can be incorporated in the soft-wall AdS/QCD model by simply modifying the 5D metric 
at the IR region. This is realized because a modification of the $5 \mathrm{D}$ metric at the IR region allows us to yield the desired IR and UV behaviors for the background dilaton field, so that the linear trajectories for the resonance meson states are simply obtained, and the resulting resonance meson states agree remarkably with the experimentally confirmed resonance states. It has also been shown that when a physically reasonable form of the bulk VEV is constructed to satisfy the boundary conditions of $A d S_{5}$, the resulting mass spectra for all the resonance meson states are not very sensitive to the choice of the exact forms of the bulk VEV, which can be seen from the tables and figures of mass spectra by comparing among three models I, II and III. While the dependence of mass spectra on the IR boundary conditions of the bulk VEV has been shown to be significant for the scalar and vector mesons, and sizable for the pseudoscalar and axial-vector mesons, which can also be seen from the tables and figures of mass spectra by comparing two cases in each model, i.e., between Ia and Ib, IIa and IIb, IIIa and IIIb, the cases $a$ and $b$ correspond to the IR boundary conditions of bulk VEV $v(z \rightarrow \infty) \sim z$ and $v(z \rightarrow \infty) \sim \sqrt{z}$, respectively. It is concluded that the case $b$ with the IR behavior $v(z \rightarrow \infty) \sim \sqrt{z}$ appears to provide more reasonable predictions for all the resonance mass spectra, which can be seen explicitly from Figs. 3 and 6 .

The effects of the quartic interaction term have been investigated in detail; a physically reasonable sign of the coupling constant for the quartic interaction, which is opposite to the one considered in [19], is taken to avoid the possible instability of the bulk scalar potential. As a consequence, it has been found that the introduction of the quartic interaction with an appropriate sign and reasonable magnitude can result in a better agreement for the resonance mass spectra of scalar, pseudoscalar, vector and axial-vector mesons. Quantitatively, the agreement with the experimental data is found to be within $10 \%$ for all resonance meson states, though the predictions for the ground state mesons of scalar, vector and axial vector are not as good as the ones for the resonance states.

As such a simply modified soft-wall AdS/QCD model can result in such remarkable predictions for the mass spectra of resonance mesons, it would be interesting to further study the dynamical origin of the metric induced conformal symmetry breaking in the IR region. Also in the present consideration, the dilaton and gravity are treated as background fields; it would also be interesting to further investigate the important role of the dilaton field in connection with the stringy features of QCD and the 5D gravity effects from the back-reacted geometry which has been studied in a class of hard-wall AdS/QCD models [31]. It is a necessity to study the possible higher order interaction terms and their effects on the mass spectra and form factors of various mesons. It is also natural to extend to the three flavor case and consider the $S U(3)$ breaking and instanton effects. 


\section{Acknowledgements}

This work was supported in part by the National Science Foundation of China (NSFC) under Grant \#No. 10821504 and the Project of Knowledge Innovation Program (PKIP) of the Chinese Academy of Science.

[1] H. Fritzsch, M. Gell-Mann and H. Leutwyler, Phys. Lett. B 47, 365 (1973).

[2] D. J. Gross and F. Wilczek, Phys. Rev. Lett. 30, 1343 (1973).

[3] H. D. Politzer, Phys. Rev. Lett. 30, 1346 (1973).

[4] Y. Nambu, Phys. Rev. Lett. 4 (1960) 380.

[5] Y. B. Dai and Y. L. Wu, Eur. Phys. J. C 39 (2005) S1 arXiv:hep-ph/0304075].

[6] G. 't Hooft, Nucl. Phys. B 72, 461 (1974).

[7] J. M. Maldacena, Adv. Theor. Math. Phys. 2, 231 (1998) [Int. J. Theor. Phys. 38, 1113 (1999)] arXiv:hep-th/9711200.

[8] S. S. Gubser, I. R. Klebanov and A. M. Polyakov, Phys. Lett. B 428, 105 (1998) arXiv:hep-th/9802109].

[9] E. Witten, Adv. Theor. Math. Phys. 2, 253 (1998) arXiv:hep-th/9802150].

[10] J. Polchinski and M. J. Strassler, Phys. Rev. Lett. 88 (2002) 031601 [arXiv:hep-th/0109174].

[11] T. Sakai and S. Sugimoto, Prog. Theor. Phys. 113, 843 (2005) arXiv:hep-th/0412141.

[12] J. Erlich, E. Katz, D. T. Son and M. A. Stephanov, Phys. Rev. Lett. 95, 261602 (2005) arXiv:hep-ph/0501128.

[13] L. Da Rold and A. Pomarol, Nucl. Phys. B 721 (2005) 79 arXiv:hep-ph/0501218.

[14] S. J. Brodsky and G. F. de Teramond, Phys. Rev. Lett. 96 (2006) 201601 arXiv:hep-ph/0602252.

[15] S. J. Brodsky and G. F. de Teramond, Phys. Rev. D 77, 056007 (2008) arXiv:0707.3859 [hep-ph]].

[16] S. J. Brodsky and G. F. de Teramond, arXiv:0909.3899 [hep-ph]; G. F. de Teramond and S. J. Brodsky, arXiv:0909.3900 [hep-ph] and references therein.

[17] A. Karch, E. Katz, D. T. Son and M. A. Stephanov, Phys. Rev. D 74, 015005 (2006) arXiv:hep-ph/0602229.

[18] P. Colangelo, F. De Fazio, F. Giannuzzi, F. Jugeau and S. Nicotri, Phys. Rev. D 78 (2008) 055009 [arXiv:0807.1054 [hep-ph]].

[19] T. Gherghetta, J. I. Kapusta and T. M. Kelley, Phys. Rev. D 79 (2009) 076003 arXiv:0902.1998 [hep-ph]].

[20] J. P. Shock and F. Wu, JHEP 0608 (2006) 023 arXiv:hep-ph/0603142].

[21] I. R. Klebanov and E. Witten, Nucl. Phys. B 556, 89 (1999) arXiv:hep-th/9905104.

[22] P. H. Damgaard and H. Fukaya, JHEP 0901, 052 (2009) [arXiv:0812.2797 [hep-lat]]. 
[23] C. Amsler et al. [Particle Data Group], Phys. Lett. B 667 (2008) 1.

[24] H. J. Kwee and R. F. Lebed, Phys. Rev. D 77, 115007 (2008) [arXiv:0712.1811 [hep-ph]].

[25] J. Polchinski and M. J. Strassler, JHEP 0305, 012 (2003) arXiv:hep-th/0209211.

[26] H. R. Grigoryan and A. V. Radyushkin, Phys. Rev. D 76, 115007 (2007) arXiv:0709.0500 [hep-ph]].

[27] V. Tadevosyan et al. [Jefferson Lab F(pi) Collaboration], Phys. Rev. C 75, 055205 (2007) arXiv:nucl-ex/0607007.

[28] T. Horn et al. [Jefferson Lab F(pi)-2 Collaboration], Phys. Rev. Lett. 97, 192001 (2006) arXiv:nucl-ex/0607005.

[29] H. Ackermann et al., Nucl. Phys. B 137, 294 (1978).

[30] P. Brauel et al., Phys. Lett. B 69, 253 (1977).

[31] J. P. Shock, F. Wu, Y. L. Wu and Z. F. Xie, JHEP 0703 (2007) 064 arXiv:hep-ph/0611227. Y. L. Wu and Z. F. Xie, JHEP 0710 (2007) 009 [arXiv:0705.2360 [hep-ph]]. 


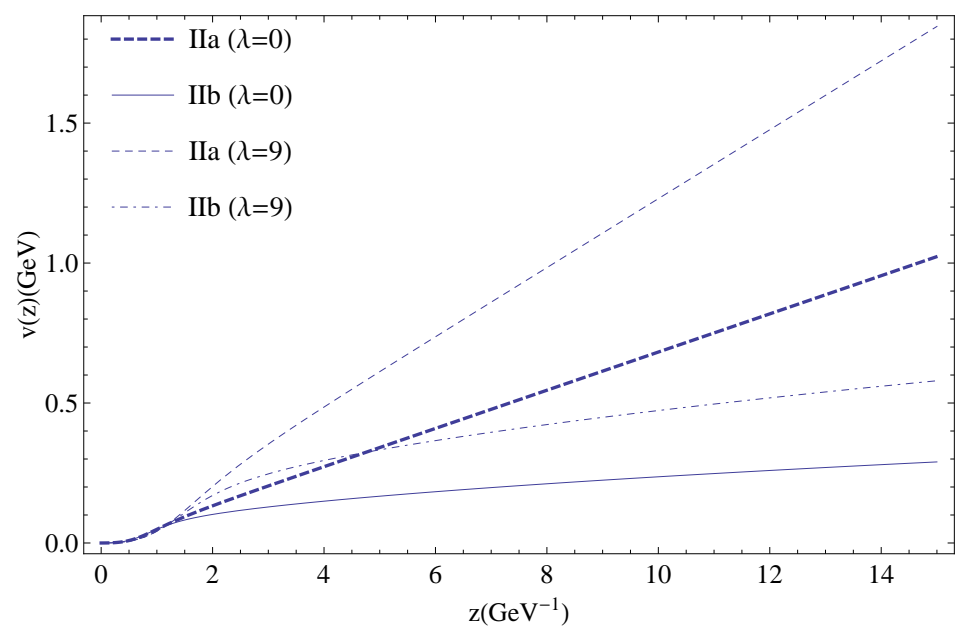

FIG. 1: A plot of $v(z)$ for various parameters which is fitted to the mass spectra.

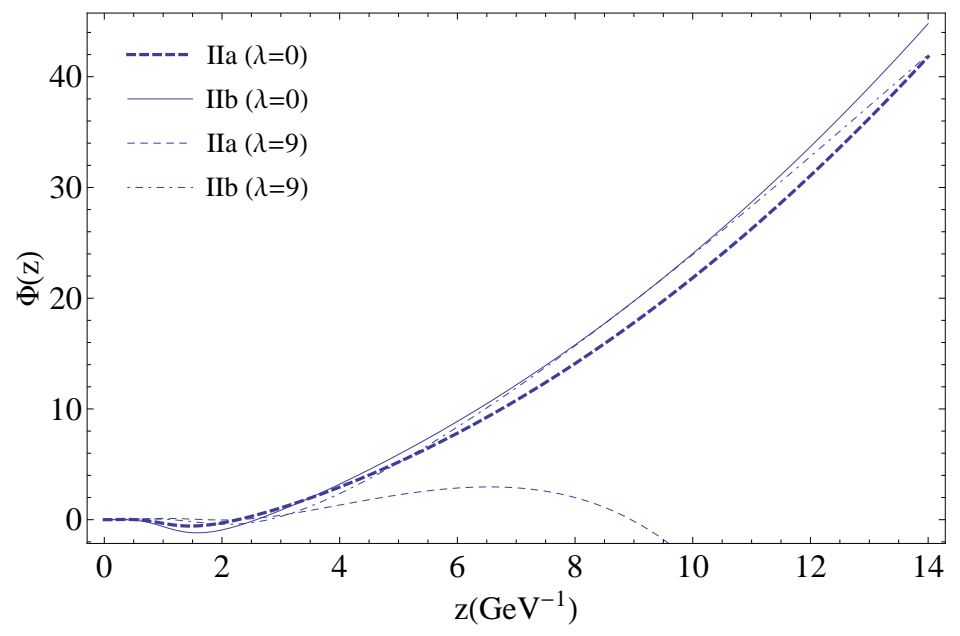

FIG. 2: A plot of dilation $\Phi(z)$ for various parameters fitted to the mass spectra 

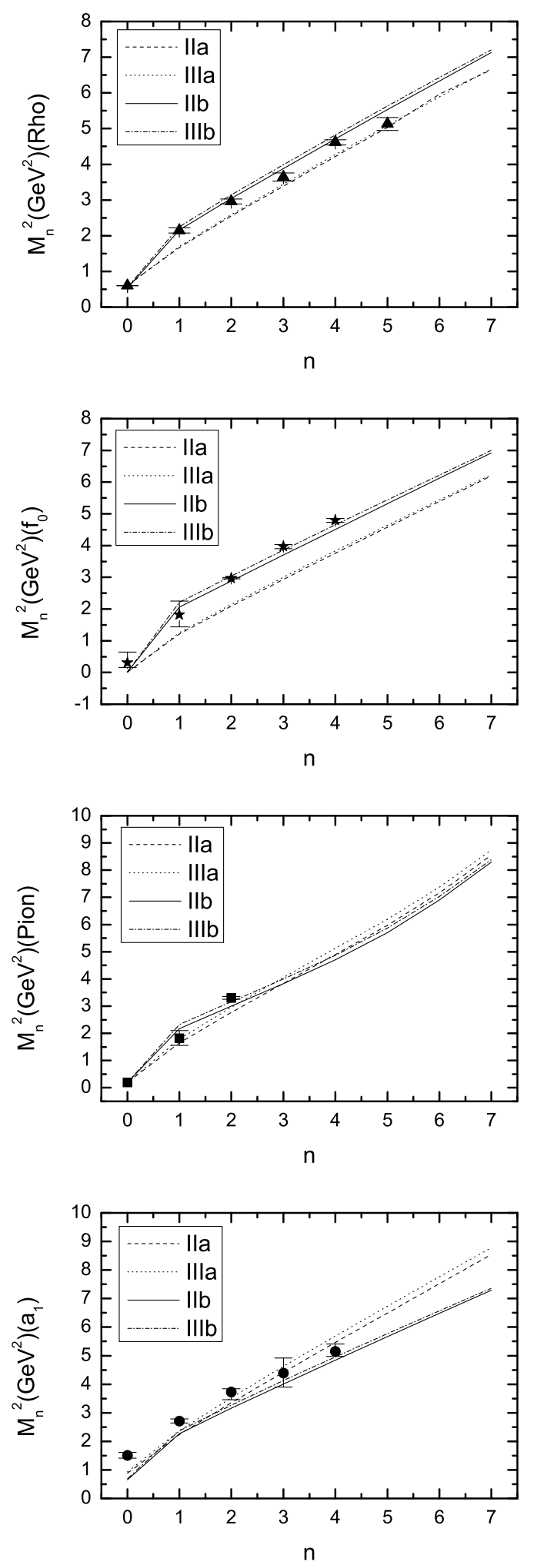

FIG. 3: A plot of mass spectra of resonance mesons in models $\operatorname{IIa}(\lambda=0), \operatorname{IIIa}(\lambda=0), \operatorname{IIb}(\lambda=0)$, $\operatorname{IIIb}(\lambda=0)$. The squares are the experimental mass spectra for the resonance pseudoscalars $(\pi)$, the triangles for the resonance vector mesons $(\rho)$, the circles for the resonance axial-vector mesons $\left(a_{1}\right)$, and the stars are for the resonance scalar mesons $\left(f_{0}\right)$. 

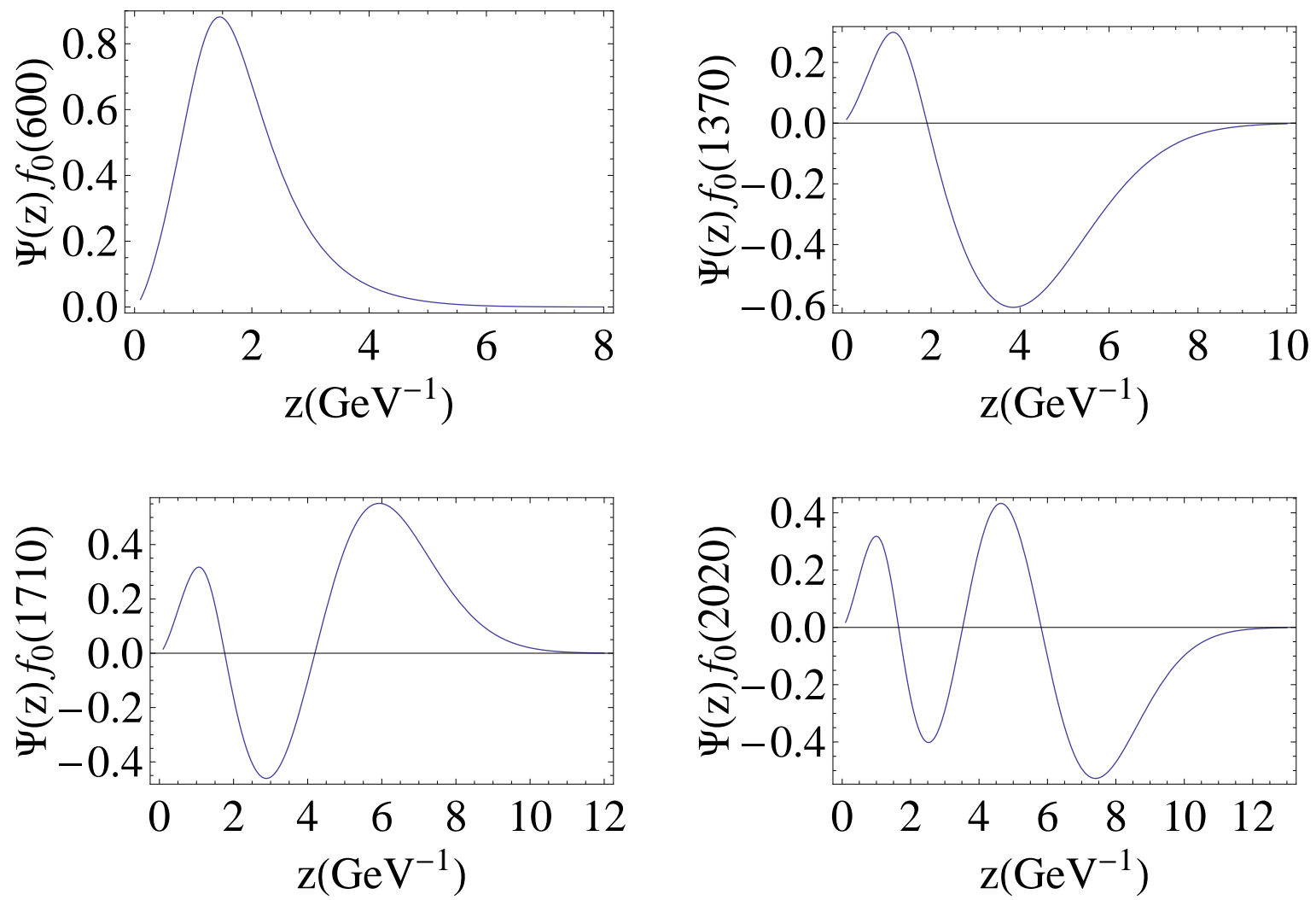

FIG. 4: Normalized bulk wave function for scalar mesons in case $\operatorname{IIb}(\lambda=0)$.

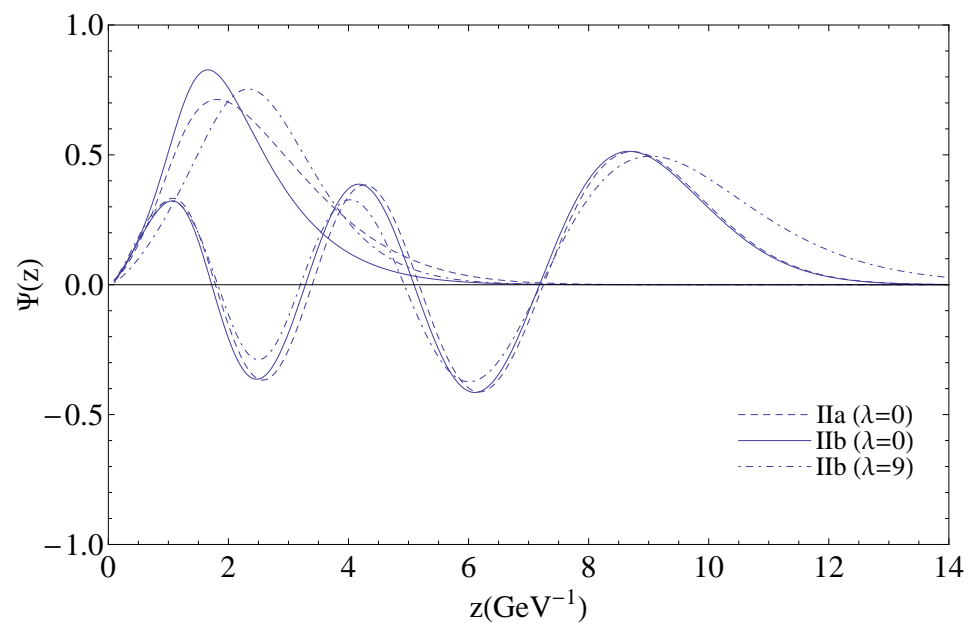

FIG. 5: Normalized bulk wave function for vector mesons with the single peak curve for the bulk wave function of ground state $\rho$ and multipeaks for the excited vector meson corresponding to $n=4$. 

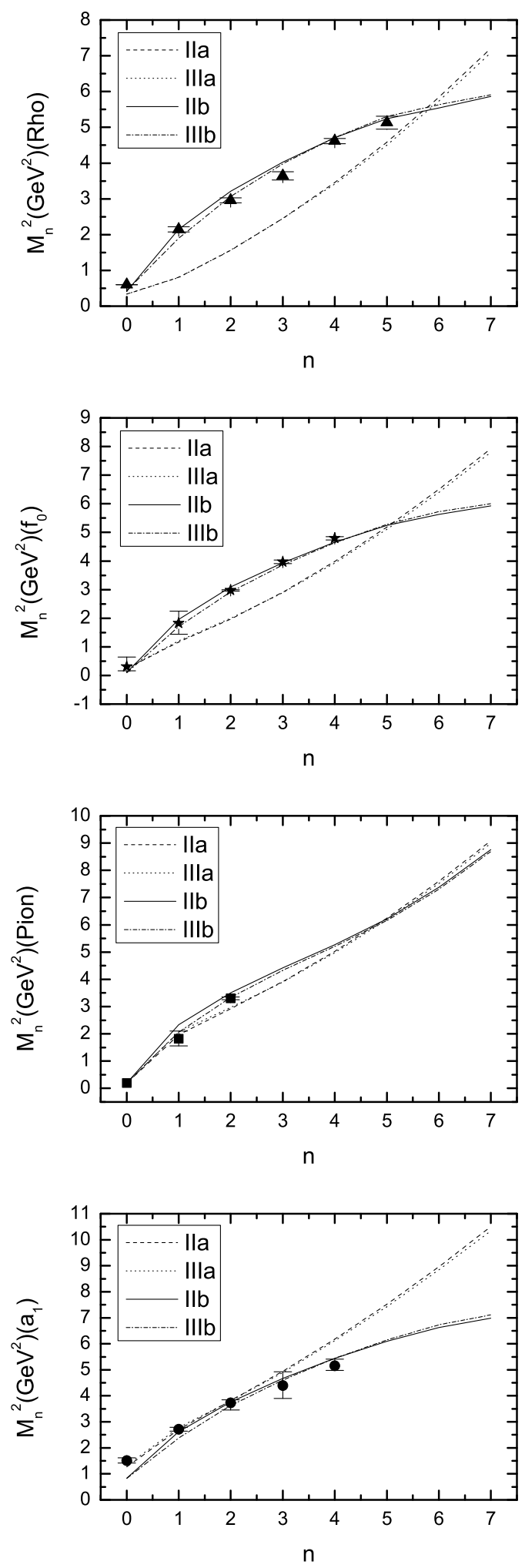

FIG. 6: Similar to Fig. 3, a plot of mass spectra of resonance mesons in models $\operatorname{IIa}(\lambda=9)$, $\operatorname{IIIa}(\lambda=9), \operatorname{IIb}(\lambda=9), \operatorname{IIIb}(\lambda=9)$. 


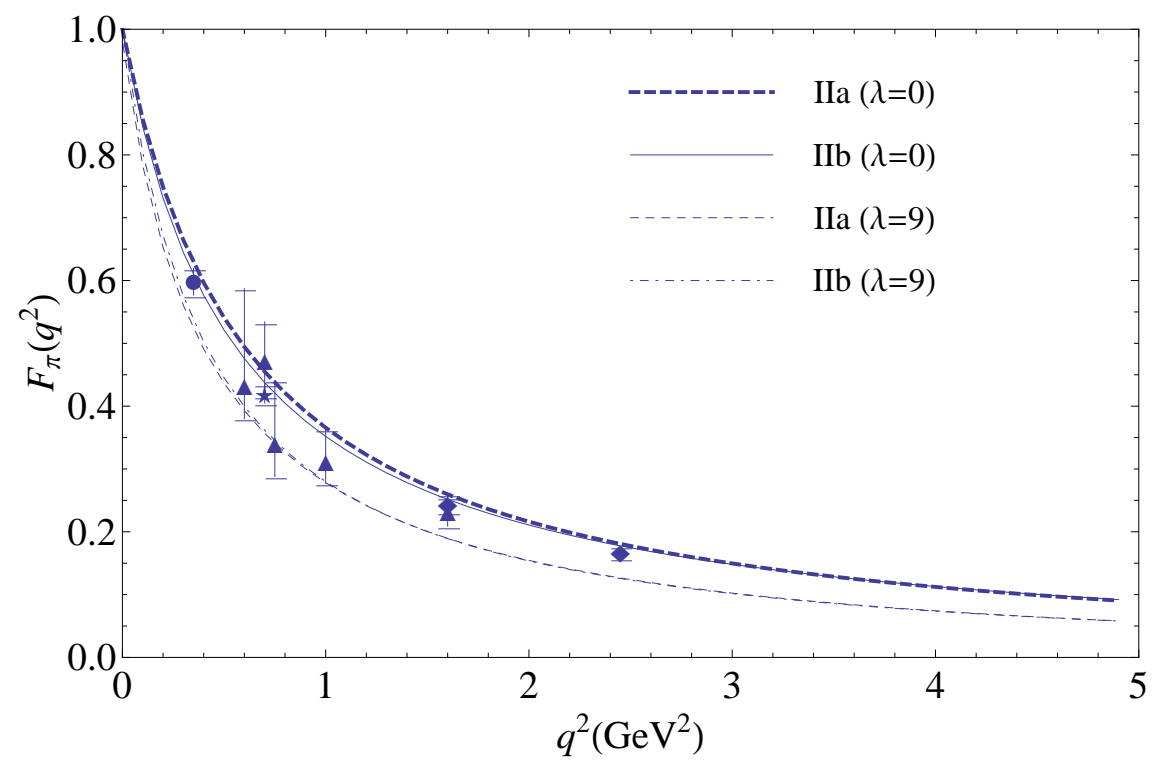

FIG. 7: The predicted spacelike behavior of the pion form factor $F_{\pi}\left(q^{2}\right)$ compared to the experimental data analyzed in [24]. The triangles are data from DESY, reanalyzed by [27]. The diamonds are data from Jefferson Lab [28]. The circle [29] as well as the star [30] are also data obtained from DESY. 